\title{
Magnetic Resonance Spectroscopic Analysis of Multifidus Muscle Lipid Contents and Association with Nociceptive Pain in Chronic Low Back Pain
}

\author{
Izaya Ogon ${ }^{1}$, Kouske Iba ${ }^{1}$, Hiroyuki Takashima ${ }^{1}$, Mitsunori Yoshimoto ${ }^{1}$, \\ Tomonori Morita ${ }^{1}$, Tsutomu Oshigiri ${ }^{1}$, Yoshinori Terashima ${ }^{1}$, Makoto Emori ${ }^{1}$, \\ Atsushi Teramoto ${ }^{1}$, Tsuneo Takebayashi ${ }^{2}$, Toshihiko Yamashita ${ }^{1}$ \\ ${ }^{1}$ Department of Orthopaedic Surgery, Sapporo Medical University School of Medicine, Sapporo, Japan \\ ${ }^{2}$ Department of Orthopaedic Surgery, Sapporo Maruyama Orthopaedic Hospital, Sapporo, Japan
}

\begin{abstract}
Study Design: Cross-sectional study.
Purpose: This study aimed to analyze the differences in the lipid contents in chronic low back pain (CLBP) patients with nociceptive pain (NocP) and neuropathic pain (NeP) using magnetic resonance spectroscopy (MRS) of the multifidus muscle (Mm).

Overview of Literature: Early identification of the pain characteristics with CLBP is important because specific treatment approaches are required, depending on NocP and NeP.

Methods: The participants were 50 patients with CLBP (23 men and 27 women; mean age, $63.1 \pm 17.8$ years; range, $41-79$ years). We compared the Visual Analog Scale (VAS) scores, intramyocellular lipids (IMCLs) and extramyocellular lipids (EMCLs) of the Mm in NocP and NeP groups, as evaluated with the Japanese NeP screening questionnaire.

Results: The patients were categorized into the NocP $(n=32)$ and NeP $(n=18)$ groups. The mean VAS score of the NocP group was $59.3 \pm 3.1 \mathrm{~mm}$ and that of the NeP group was $73.6 \pm 4.6 \mathrm{~mm}$. The mean VAS score was significantly higher in the NeP group as compared to that in the NocP group $(p<0.01)$. As per the analysis of covariance for the VAS score, the mean IMCL levels of the Mm in the NocP and NeP groups were $722.3 \mathrm{mmol} / \mathrm{L}$ (95\% confidence interval [Cl], 611.4-833.1) and $484.8 \mathrm{mmol} / \mathrm{L}(95 \% \mathrm{Cl}, 381.1-588.5)$, respectively. The mean IMCL level was significantly higher in the NocP group than in the NeP group $(p<0.05)$. The mean EMCL levels of the $\mathrm{Mm}$ for the NocP and NeP groups were 6,022.9 mmol/L (95\% Cl, 4,510.6-7,535.2) and 5,558.1 $\mathrm{mmol} / \mathrm{L}(95 \% \mathrm{Cl}, 4,298.3-6,817.9)$, respectively; however, the difference was not significant $(p=0.72)$.

Conclusions: The results indicated an association between the IMCL level of the Mm and NocP. Our results suggest that MRS of the Mm might be beneficial for the assessment of CLBP as well as appropriate targeted analgesic therapies.
\end{abstract}

Keywords: Low back pains; Magnetic resonance imaging; Paraspinal muscle; Nociceptive pains

\section{Introduction}

Low back pain (LBP) is one of the most common physi- ological issues worldwide [1]. Recently, Suzuki et al. [2] reported that $78 \%$ of patients with LBP were diagnosed based on the data collected using questionnaires, physical

\footnotetext{
Received May 18, 2020; Revised Jun 29, 2020; Accepted Jul 4, 2020

Corresponding author: Izaya Ogon

Department of Orthopaedic Surgery, Sapporo Medical University School of Medicine, 291, Soutn-1, West-16, Chuo-ku, Sapporo, 0608543, Japan

Tel: +81-11-611-2111, Fax: +81-11-641-6026, E-mail: ogon.izaya@sapmed.ac.jp
} 
findings, and the various block injections. In particular, disorders of the multifidus muscle $(\mathrm{Mm})$ that plays an important role in trunk function [3] have been associated with chronic low back pain (CLBP) [4].

Pain symptoms, including LBP, were classified into two broad mechanism-based pain categories as follows: nociceptive pain (NocP) that is caused by tissue-injury and/or inflammation and neuropathic pain $(\mathrm{NeP})$ that is caused by nervous system injury [5,6]. A different treatment approach is required for each pain category [7]; therefore, early identification of the involvement of NocP and $\mathrm{NeP}$ in patients with chronic pain is important for effective pain management [8].

Recently, several studies have reported on the quantitative evaluation of $\mathrm{Mm}$ fatty degeneration with magnetic resonance spectroscopy (MRS) [9-16]. MRS analysis of muscle physiology has facilitated detailed analyses of muscular fat masses via the identification of intramyocellular lipids (IMCLs) and extramyocellular lipids (EMCLs) that has enabled detailed assessment of fatty degeneration $[9,10]$. IMCLs cannot be detected using conventional magnetic resonance imaging (MRI) because they appear as lipid droplets in the skeletal muscle cells that are in close contact with skeletal mitochondria [10,11]. In contrast, EMCLs represent subcutaneous and extracellular fat in the cells between the muscle fibers $[9,17,18]$. In our previous study, MRS analyses of the Mm showed that CLBP patients had significantly higher IMCL levels than controls $[12,15,16]$.

This study aimed to analyze the differences in the lipid contents in NocP and NeP by performing MRS of the Mm in CLBP patients.

\section{Materials and Methods}

The institutional review board of the Sapporo Medical
University approved this study (IRB approval no., 2621074). All the participants were provided with written and verbal explanations of the study procedure, and all of them provided their consent before participating.

\section{Participants}

Participants were patients (range, 41-79 years old) with non-specific CLBP, characterized by pain, stiffness, and discomfort in the lower back from the 12th rib to the lumbar area or the lumbosacral area, wherein the source of the pain was difficult to identify. Furthermore, the symptoms of all participants had persisted for more than 3 months despite conservative treatments, such as medication and therapeutic exercise. As per the exclusion criteria, we did not enroll patients with neoplasm, infection, fracture, history of lumbar vertebral surgery, neurologic symptoms of the lower leg, or obvious instability that could be the source of LBP and might be improved with surgical treatment. LBP was assessed using the Visual Analog Scale (VAS, 0-100) and the Japanese NeP screening questionnaire after a washout period of at least 4 weeks [19] (Table 1). The Japanese NeP screening questionnaire categorizes patients with a score $<4$ as having NocP, while those with a score $\geq 4$ were characterized as having NeP.

\section{Magnetic resonance imaging protocol and analysis for magnetic resonance spectroscopic data}

We used the previously described MRI protocol and methods of analysis for magnetic resonance (MR) spectroscopic data [12-16]. In brief, the Signa HDx 1.5-T MRI system (GE Healthcare, Milwaukee, WI, USA) with a spine coil was employed to obtain T2 weighted sagittal and transverse MR images. From these images, the proton MRS volume of interest (VOI) was positioned in the

Table 1. Questions on the nature of pain

\begin{tabular}{|c|c|c|c|c|c|}
\hline Question & None & Slight & Moderate & Severe & Very severe \\
\hline 01. Pinprick-like pain & 1 & 1 & 1 & 1 & 0 \\
\hline 02. Electric shock-like pain & 0 & 0 & 0 & 0 & 1 \\
\hline 03. Tingling burning pain & 0 & 1 & 1 & 1 & 1 \\
\hline 04. Pain with strong numbness & 0 & 1 & 1 & 1 & 1 \\
\hline 05. Only a light touch with clothing or cold wind causes a pain. & 0 & 1 & 3 & 3 & 3 \\
\hline Q6. Site of pain has decreased or increased sensation. & 0 & 1 & 1 & 1 & 1 \\
\hline 07. Site of pain shows skin swelling and/or discoloration to red or purple. & 0 & 0 & 0 & 1 & 1 \\
\hline
\end{tabular}


center of the Mm at L4/L5 on the right side (Fig. 1). The single voxel point-resolved spectroscopy sequence was performed with the following parameters: repetition time, 2,000 ms; echo time, $35 \mathrm{~ms}$; average number of signals, 64 ; VOI size, $15 \times 15 \times 15 \mathrm{~mm}(3.4 \mathrm{~mL})$; and acquisition time, 164 seconds.

The recorded spectral data were used to measure the levels of IMCLs and EMCLs using the LCModel software (Stephen Provencher Inc., Oakville, ON, USA) [20]. Data

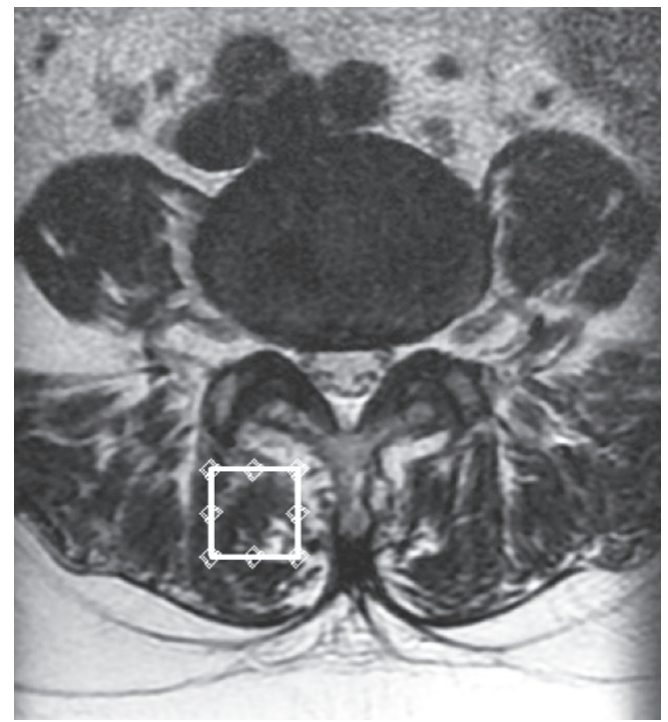

Fig. 1. Volume of interest for magnetic resonance spectroscopy measurements of the right multifidus muscle as indicated on the T2 weighted-image at the L4/5 level.

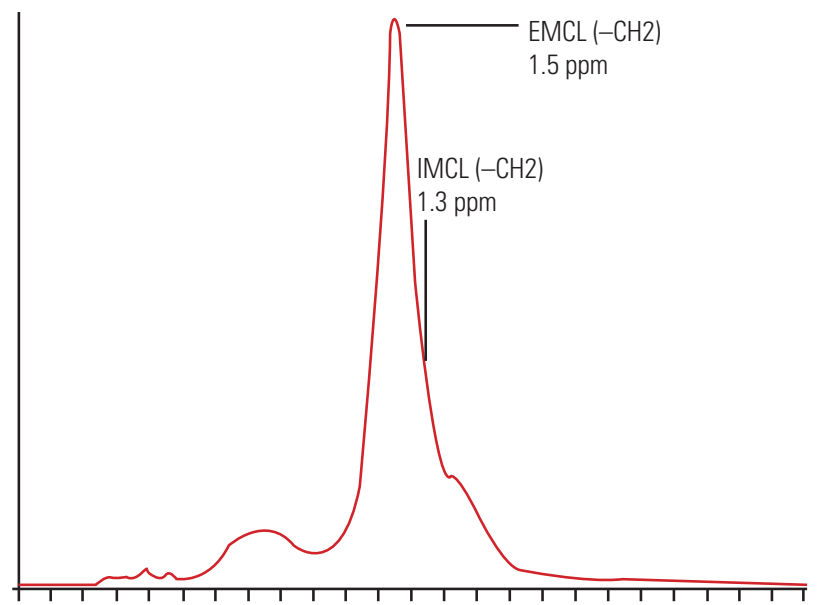

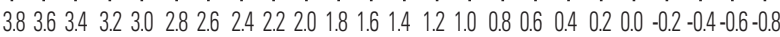
Chemical shift (ppm)

Fig. 2. Proton magnetic resonance spectrum of multifidus muscle analyzed using LCModel software (Stephen Provencher Inc., Oakville, ON, USA). The following metabolites are identified; IMCLs (-CH2) methylene protons at $1.3 \mathrm{ppm}$; EMCLs (-CH2) methylene protons at $1.5 \mathrm{ppm}$. IMCLs, intramyocellular lipids; EMCLs, extramyocellular lipids. were transferred from the scanners to a Linux workstation, and metabolite quantification was performed with Eddy current correction and water scaling. The methylene signals of IMCLs (1.3 ppm) and EMCLs (1.5 ppm) were used for quantification. Estimates of IMCL and EMCL concentrations were automatically scaled to an unsuppressed water peak $(4.7 \mathrm{ppm})$ and the total creatine peak (approximately $3.03 \mathrm{ppm}$ ) to determine the lipid concentrations in $\mathrm{mmol} / \mathrm{L}[21,22]$. A typical MR spectrum is presented in Fig. 2. Participants with Mm IMCLs or EMCLs content $>15 \%$ above the standard deviation were excluded from the LCModel analysis.

\section{Statistical analyses}

Demographic data regarding sex-distribution were analyzed by the chi-square test. Differences among the groups were compared using the Mann-Whitney $U$-test. We compared the IMCLs and EMCLs of the Mm in the NocP and $\mathrm{NeP}$ groups using analysis of covariance (ANCOVA) as covariance of the VAS score. A $p$-value $<0.05$ indicated statistical significance. Numerical data are expressed as mean \pm standard error of the mean or mean $(95 \%$ confidence interval $[\mathrm{CI}])$.

\section{Results}

In total, 50 patients ( 23 males, 27 females; mean age, $63.1 \pm 17.8$ years; range, $41-79$ years) met the diagnostic criteria. The mean body mass index (BMI) was $23.7 \pm 0.5$ $\mathrm{kg} / \mathrm{m}^{2}$, and the mean VAS score was $65.3 \pm 2.9 \mathrm{~mm}$.

As shown in Table 2 and Fig. 3, the patients were categorized as having $\operatorname{NocP}(n=32)$ or NeP $(n=18)$. The patients had an almost equal number of men and women, with no significant differences $(p=0.87)$. There were no significant differences in the mean age and BMI of the two groups ( $p=0.81$ and $p=0.83$ ), respectively. The average values of the NeP scores were $2.1 \pm 0.1$ and $5.6 \pm 0.4$ in the $\mathrm{NocP}$ and NeP groups, respectively. The mean VAS score of the NocP group was $59.3 \pm 3.1 \mathrm{~mm}$ and that of the NeP group was $73.6 \pm 4.6 \mathrm{~mm}$ (Fig. 4). The mean VAS score was significantly higher in the $\mathrm{NeP}$ as compared to that in the NocP group $(p<0.01)$.

According to ANCOVA as covariance of the VAS score, the mean IMCLs of the $\mathrm{Mm}$ in the NocP and NeP groups were $722.3 \mathrm{mmol} / \mathrm{L}$ (95\% CI, 611.4-833.1), and 484.8 $\mathrm{mmol} / \mathrm{L}$ (95\% CI, 381.1-588.5), respectively. The mean 
IMCLs was significantly higher in the NocP group than in the NeP group $(p<0.05)$ (Fig. 5A). The mean EMCLs of the $\mathrm{Mm}$ for the NocP and NeP groups were $6,022.9 \mathrm{mmol} /$ L (95\% CI, 4,510.6-7,535.2) and 5,558.1 mmol/L (95\% CI, 4,298.3-6,817.9), respectively; however, the difference was not significant $(p=0.72)$ (Fig. $5 \mathrm{~B})$.

\section{Discussion}

Non-specific LBP is estimated to account for $>85 \%$ of all LBP cases [1]. Mm is considered a cause of pain onset for LBP [4]; however, the detail of pain remains unclear. The diagnosis of NocP and NeP components as the cause of LBP is difficult because a gold standard examination is lacking. The diagnostic value of clinical interviews, physical examinations and imaging analyses has not been well documented. Previous reports have shown that NocP is underdiagnosed and undertreated [23]. Thus, no specific

Table 2. Patient demographic data concerning the gender, age, NeP, and VAS score

\begin{tabular}{lrrr} 
Characteristic & NocP $(\mathrm{n}=32)$ & $\mathrm{NeP}(\mathrm{n}=18)$ & $p$-value \\
\hline Gender (male:female) & $15: 17$ & $8: 10$ & $0.87^{\mathrm{a})}$ \\
\hline Age $(\mathrm{yr})$ & $63.3 \pm 2.0$ & $62.5 \pm 2.6$ & $0.81^{\mathrm{b}}$ \\
\hline Body mass index $\left(\mathrm{kg} / \mathrm{m}^{2}\right)$ & $24.0 \pm 0.7$ & $23.5 \pm 0.7$ & $0.83^{\mathrm{b}}$ \\
\hline NeP score $($ points & $2.0 \pm 0.1$ & $5.6 \pm 0.4$ & $<0.01^{\mathrm{b}}$ \\
\hline VAS score $(\mathrm{mm})$ & $59.3 \pm 3.1$ & $73.6 \pm 4.6$ & $<0.01^{\mathrm{b}}$ \\
\hline
\end{tabular}

Values are presented as number or mean \pm standard error of the mean. NocP, nociceptive pain; NeP, neuropathic pain; VAS, visual analog scale. alBy chi-square test. ${ }^{\text {b/B }}$ By Mann-Whitney U-test.

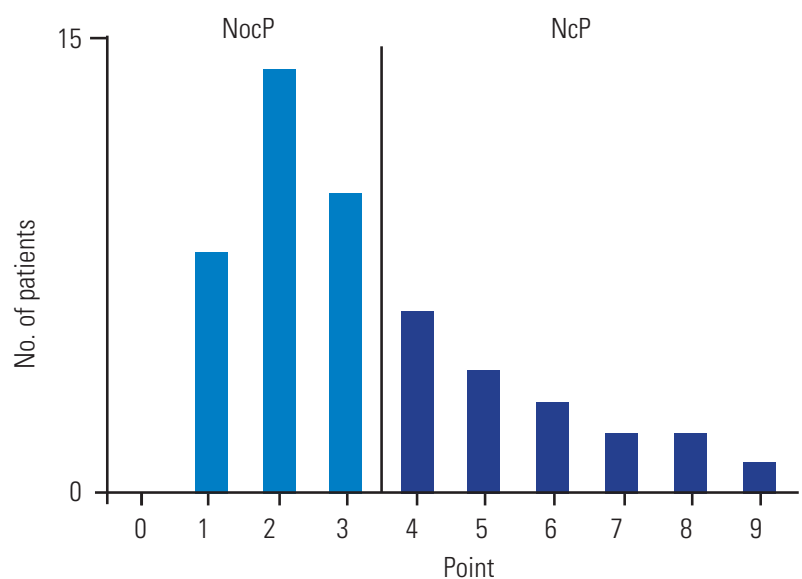

Fig. 3. Bar chart of the number of patients in each neuropathic pain (NeP) score. Patients were categorized into the nociceptive pain (NocP) group ( $n=32$ ) and the $\mathrm{NeP}(\mathrm{n}=18)$ group. diagnostic method has been established, and diagnosing and treating this condition remains a challenge.

In previous studies, the adipose tissue of the $\mathrm{Mm}$ was separated into IMCLs and EMCLs, and we identified an association between the levels of IMCLs and LBP $[12,15,16]$. In the present study, we used MRS of the Mm in CLBP in order to quantitatively investigate the differences between NocP and NeP, revealing the correlations between IMCLs, VAS scores, NocP, and NeP. There was an association between the IMCL levels and VAS scores. Therefore, it is possible that the mitochondrial activity of the Mm is impaired, resulting in an increased level of inflammatory cytokines, such as interleukin-6 (IL-6) and tumor necrosis factor- $\alpha$ (TNF- $\alpha$ ) in the Mm of patients with CLBP [24,25]. Tesarz et al. [26] reported that the

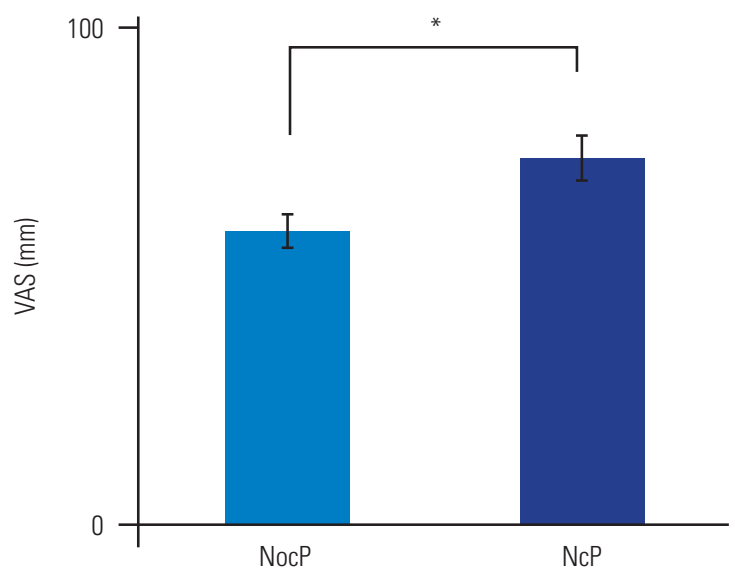

Fig. 4. Bar chart showing Visual Analog Scale (VAS) scores in the nociceptive pain (NocP) and neuropathic pain (NeP) groups. VAS scores were significantly higher in the NeP than in the NocP group $(p<0.01)$. Error bars denote the standard error of the mean. ${ }^{*}<0.01$ (by the Mann-Whitney $U$-test).
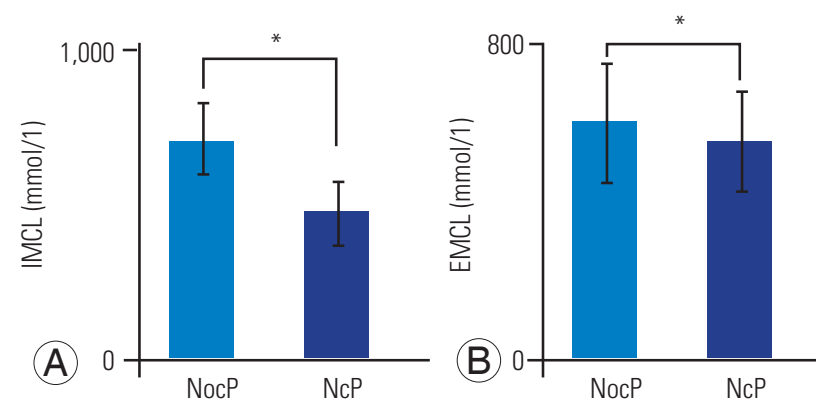

Fig. 5. Bar chart showing intramyocellular lipids (IMCLs) (A) and extramyocellular lipids (EMCLs) (B) of the multifidus muscle in the nociceptive pain (NocP) and neuropathic pain (NeP) groups. The mean IMCLs was significantly higher in the NocP than in the NeP group. The mean EMCLs was not significantly different between the NocP and NeP groups. Error bars denote the $95 \%$ confidence interval. ${ }^{*} p<0.05$ (by the analysis of covariance as covariance of Visual Analog Scale score). 
thoracolumbar fascia is a densely innervated tissue with marked differences in the distribution of nerve endings throughout the fascial layers. Sensory nerve innervation of the thoracolumbar fascia and increases in the levels of cytokines, such as IL- 6 and TNF- $\alpha$, associated with IMCLs in the Mm might be the irrelevant NocP pathophysiology.

This study has important implications for targeted analgesic therapies in clinical settings. A correct understanding of NocP and NeP in CLBP and suitable treatments might enable more effective and patient-oriented pain control. The present results suggest that the IMCL level in the Mm could help in the assessment of CLBP as well as appropriate targeted analgesic therapies.

This study has certain limitations. First, the sample size was relatively small, and there was no control group. Second, the Japanese $\mathrm{NeP}$ screening questionnaire might be an inadequate diagnostic tool for detecting $\mathrm{NeP}$ caused by spinal disorders. As per a nationwide study in Japan using the $\mathrm{NeP}$ screening questionnaire, higher pain scores were associated with higher $\mathrm{NeP}$ rates [27]. It has been suggested that the mechanism, nature, and intensity of pain should have been independent factors; however, these might be confounding factors that lead to an increase in apparent pain intensity and give a false high $\mathrm{NeP}$ score. Therefore, we conducted ANCOVA analyses to exclude confounding with the VAS score.

\section{Conclusions}

Our results indicate an association between the IMCLs of the Mm and NocP. This study suggests that MRS of the Mm might enable the assessment of CLBP as well as appropriate targeted analgesic therapies.

\section{Conflict of Interest}

No potential conflict of interest relevant to this article was reported.

\section{Acknowledgments}

This work was supported by the Japan Society for the Promotion of Science KAKENHI (Grant no., 18K16667).

\section{References}

1. Deyo RA, Weinstein JN. Low back pain. N Engl J Med 2001;344:363-70.

2. Suzuki H, Kanchiku T, Imajo Y, Yoshida Y, Nishida N, Taguchi T. Diagnosis and characters of non-specific low back pain in Japan: the Yamaguchi low back pain study. PLoS One 2016;11:e0160454.

3. Wilke HJ, Wolf S, Claes LE, Arand M, Wiesend A. Stability increase of the lumbar spine with different muscle groups: a biomechanical in vitro study. Spine (Phila Pa 1976) 1995;20:192-8.

4. Ranger TA, Cicuttini FM, Jensen TS, et al. Are the size and composition of the paraspinal muscles associated with low back pain?: a systematic review. Spine J 2017;17:1729-48.

5. Woolf CJ, Bennett GJ, Doherty M, et al. Towards a mechanism-based classification of pain? Pain 1998;77:227-9.

6. Freynhagen R, Baron R, Tolle T, et al. Screening of neuropathic pain components in patients with chronic back pain associated with nerve root compression: a prospective observational pilot study (MIPORT). Curr Med Res Opin 2006;22:529-37.

7. Baron R, Binder A, Wasner G. Neuropathic pain: diagnosis, pathophysiological mechanisms, and treatment. Lancet Neurol 2010;9:807-19.

8. Haanpaa ML, Backonja MM, Bennett MI, et al. Assessment of neuropathic pain in primary care. Am J Med 2009;122(10 Suppl):S13-21.

9. Boesch C, Machann J, Vermathen P, Schick F. Role of proton MR for the study of muscle lipid metabolism. NMR Biomed 2006;19:968-88.

10. Boesch C. Musculoskeletal spectroscopy. J Magn Reson Imaging 2007;25:321-38.

11. Boesch C, Kreis R. Observation of intramyocellular lipids by $1 \mathrm{H}$-magnetic resonance spectroscopy. Ann N Y Acad Sci 2000;904:25-31.

12. Ogon I, Takebayashi T, Takashima H, et al. Magnetic resonance spectroscopic analysis of multifidus muscles lipid content and association with spinopelvic malalignment in chronic low back pain. Br J Radiol 2017;90:20160753.

13. Ogon I, Takebayashi T, Takashima H, et al. Multifidus muscles lipid content is associated with intervertebral disc degeneration: a quantitative magnetic resonance imaging study. Asian Spine J 2019;13:601-7. 
14. Ogon I, Takebayashi T, Takashima H, et al. Quantitative analysis concerning atrophy and fat infiltration of the multifidus muscle with magnetic resonance spectroscopy in chronic low back pain. Spine Surg Relat Res 2018;3:163-70.

15. Takashima H, Takebayashi T, Ogon I, et al. Evaluation of intramyocellular and extramyocellular lipids in the paraspinal muscle in patients with chronic low back pain using MR spectroscopy: preliminary results. Br J Radiol 2016;89:20160136.

16. Takashima H, Takebayashi T, Ogon I, et al. Analysis of intra and extramyocellular lipids in the multifidus muscle in patients with chronic low back pain using MR spectroscopy. Br J Radiol 2018;91:20170536.

17. Velan SS, Said N, Durst C, et al. Distinct patterns of fat metabolism in skeletal muscle of normal-weight, overweight, and obese humans. Am J Physiol Regul Integr Comp Physiol 2008;295:R1060-5.

18. Srikanthan P, Singhal A, Lee CC, et al. Characterization of intra-myocellular lipids using 2D localized correlated spectroscopy and abdominal fat using MRI in type 2 diabetes. Magn Reson Insights 2012;5:29-36.

19. Ogawa S. Development of new screening questionnaire to identify neuropathic components in Japanese patients with chronic pain. Pain Clinic 2010;31:118794.
20. Provencher SW. Estimation of metabolite concentrations from localized in vivo proton NMR spectra. Magn Reson Med 1993;30:672-9.

21. Torriani M, Thomas BJ, Halpern EF, Jensen ME, Rosenthal DI, Palmer WE. Intramyocellular lipid quantification: repeatability with $1 \mathrm{H}$ MR spectroscopy. Radiology 2005;236:609-14.

22. Weis J, Johansson L, Ortiz-Nieto F, Ahlstrom H. Assessment of lipids in skeletal muscle by LCModel and AMARES. J Magn Reson Imaging 2009;30:1124-9.

23. Taylor RS. Epidemiology of refractory neuropathic pain. Pain Pract 2006;6:22-6.

24. Dyck DJ, Heigenhauser GJ, Bruce CR. The role of adipokines as regulators of skeletal muscle fatty acid metabolism and insulin sensitivity. Acta Physiol (Oxf) 2006;186:5-16.

25. Amouzou C, Breuker C, Fabre O, et al. Skeletal muscle insulin resistance and absence of inflammation characterize insulin-resistant grade I obese women. PLoS One 2016;11:e0154119.

26. Tesarz J, Hoheisel U, Wiedenhofer B, Mense S. Sensory innervation of the thoracolumbar fascia in rats and humans. Neuroscience 2011;194:302-8.

27. Yamashita T, Takahashi K, Yonenobu K, Kikuchi S. Prevalence of neuropathic pain in cases with chronic pain related to spinal disorders. J Orthop Sci 2014;19:15-21. 